\title{
Model versus non-model based left ventricular volumetry - A matter of imaging modality or quantification software?
}

\author{
Kai Thorsten Laser ${ }^{1}$, Peter Barth ${ }^{2}$, Miriam Bunge ${ }^{1}$, Gregor Dachner ${ }^{2}$, Hermann Esdorn ${ }^{2}$, Marcus \\ Fischer $^{1}$, Jürgen Gieseke ${ }^{3}$, Eugen Sandica ${ }^{4}$, Deniz Kececioglu ${ }^{1}$, Wolfgang Burchert ${ }^{2}$, Hermann \\ Körperich ${ }^{2}$
}

1. Center for Congenital Heart Defects, Heart and Diabetes Center Northrhine-Westfalia, Ruhr-University of Bochum, Georgstrasse, Bad Oeynhausen, Germany. 2. Institute for Radiology, Nuclear Medicine and Molecular Imaging, Heart and Diabetes Center Northrhine-Westfalia, Ruhr-University of Bochum, Georgstrasse, Bad Oeynhausen, Germany. 3. MR Clinical Science, Philips Healthcare, The Netherlands. 4. Department of Surgery for Congenital Heart Defects, Heart and Diabetes Center Northrhine-Westfalia, Ruhr-University of Bochum, Georgstrasse, Bad Oeynhausen, Germany.

Correspondence: Kai Thorsten Laser. Address: Center for Congenital Heart Defects, Heart and Diabetes Center Northrhine-Westfalia, Ruhr-University of Bochum, Georgstrasse 11, D-32545 Bad Oeynhausen, Germany. Telephone: 49-5731-97-3637. Email: tlaser@hdz-nrw.de.

Received: September 1, 2012

DOI : $10.5430 / j b g c . v 3 n 2 p 54$
Accepted: December 5, 2012

URL: http://dx.doi.org/10.5430/jbgc.v3n2p54

\section{Abstract}

Objective: To compare the modalities 3D-echocardiography (RT3DE) and cardiac magnetic resonance as well as semiautomatic non-model-based and model-based quantification software (SWP-MRI and SWT-MRI) regarding accuracy and agreement of left ventricular functional indices.

Methods: 9 asymmetrically shaped gel phantoms (range: $20-350 \mathrm{ml}), 24$ healthy children (age $=11.4 \pm 3.3 \mathrm{y}$ ) and 11 patients with abnormally shaped left ventricles $(22.0 \pm 17.0 \mathrm{y})$ were prospectively investigated. 3D-echocardiography was performed using a Vivid 7 ultrasound machine (V3 matrix transducer); postprocessing was done with a model-based analysis strategy (SWT-echo). CMR datasets were obtained using a multi-slice multi-phase steady-state-free-precision acquisition (TR/TE/flip $=2.8 \mathrm{msecs} / 1.4 \mathrm{msecs} / 60^{\circ}$ ) with a $1.5 \mathrm{~T} \mathrm{MR} \mathrm{system.} \mathrm{Volume} \mathrm{quantification} \mathrm{was} \mathrm{done} \mathrm{using} \mathrm{the} \mathrm{same}$ model-based software for CMR as well as non model-based software based on the summation of discs method. Agreement of EDV, ESV and EF between SWT-echo, SWP-mri vs. SWT-mri was determined by Bland Altman analysis.

Results: Phantom study revealed high accuracy $(<1 \%)$ for SWT-echo and SWP-mri as well as a moderate underestimation for SWT-MRI (13\%). Agreement between SWP-mri and SWT-echo was superior in volunteers [mean; limits-ofagreement: $\operatorname{EDV}(5.3 \%$; -20.1 to $30.8 \%)$, $\operatorname{ESV}(-1.3 \%$; -41.6 to $38.9 \%), \operatorname{EF}(4.0 \%$; -12.0 to $19.9 \%)$ ] with only slight underestimation by RT3DE in patients [EDV(11.5\%; -18.5 to $41.4 \%), \operatorname{ESV}(13.0 \% ;-5.4$ to $31.5 \%), \operatorname{EF}(-6.9 \% ;-49.9$ to $36.1 \%)]$. Comparing SWT-echo with SWT-mri revealed volume underestimation of EDV $(9.8 ;-20.5$ to $40.0 \%)$ and overestimation of ESV $(-9.6 ;-60.1$ to $41.0 \%)$ in volunteers by SWT-mri resulting in underestimation of EF (12.6;-9.6 to 34.9). In patients minor differences between SWT-echo and SWT-mri were observed [EDV (0.6\%; -28.2 to 29.4\%), ESV $(-2.4 \% ;-38.2$ to $33.4 \%), \mathrm{EF}(9.3 \% ;-35.7$ to $54.3 \%)]$. Compared to our reference SWP-mri both model-based techniques moderately underestimated EDV (SWT-MRI $12.1 \%$; -2.1 to $26.4 \%$, SWT-echo $11.5 \%$; -18.5 to $41.4 \%$ ) and ESV 
(SWT-MRI $10.6 \%$; -21.2 to $42.4 \%$, SWT-echo $13.0 \%$; -5.4 to $31.5 \%$ ) resulting in quite precise EF(SWT-MRI $2.4 \%$, -23.7 to $28.5 \%$ ).

Conclusion: Accuracy and reliability of left ventricular indices are excellent for RT3DE assessed by model based approach compared to non-model-based CMR approach in phantoms and healthy volunteers with minor volume underestimation in atypically shaped moving ventricles. Minor agreement was present if the model-based CMR software was used for determination of ventricular volumes.

\section{Key words}

Magnetic resonance imaging, Three-dimensional echocardiography, Left ventricular function, Pediatrics, Quantitative evaluation

\section{Background}

Accurate assessment of left ventricular (LV) indices is indispensable for children with cardiac disease having diagnostic and prognostic implications ${ }^{[1]}$. This is especially important for the decision making in small borderline ventricles as well as in dilated ventricles because of volume overload regarding the kind and timing of further treatment. Most non-invasive imaging modalities such as 2D-Echocardiography are using geometric assumptions and therefor lack accuracy in the assessment of the patients with abnormally shaped ventricles ${ }^{[2]}$. Currently, cardiovascular magnetic resonance imaging (CMR) is regarded as the first line imaging technique for the evaluation of ventricular volumes, mass and function both in normally shaped as well as in abnormally shaped ventricles as stated by a consensus panel report ${ }^{[3]}$ because of its high accuracy, reproducibility and independence of geometric assumptions ${ }^{[4]}$. With the introduction of second generation matrix transducers and new postprocessing software RT3DE has approached accuracy and reproducibility of CMR especially for LV volumetry ${ }^{[5,6]}$ being a good alternative because of less acquisition time and no need for sedation even in small children in contrast to CMR. A variety of different contour-tracking techniques using semiautomatic segmentation of the LV cavity for postprocessing of RT3DE and CMR ${ }^{[7-13]}$ have been proposed. Especially RT3DE tools provide a timesaving workflow generating volumes as well as volume-time curves in order to assess dyssynchrony and regional function. They use model-dependent contour tracking algorithms or speckle-tracking based approaches ${ }^{[7,14-16]}$. In summary they provide reliable and reproducible data about LV-EF with mild or moderate underestimation of volumes. This happens although papillary muscles are separated from the muscle and are calculated as volume of the cavity emphasizing the significance of contour finding algorithms in the trabeculated endocardium. In the field of CMR most of the quantification tools are using non-model based and threshold based two-dimensional (2D) ${ }^{[11,17,18]}$ or threedimensional (3D) ${ }^{[8,9,19]}$ segmentation approaches. Only few of them are capable to derive LV volume-time curves ${ }^{[12,20]}$. Furthermore, postprocessing for correct manual delineation of chamber boundaries is time consuming and operator dependent leading to a higher intraobserver and interobserver variability and thus to a poorer clinical value. On the other hand trabecular structures as well as papillary muscles can be addressed more precisely and are calculated as muscular structures. To improve workflow and to keep time demand acceptable for clinical routine model-dependent contour tracking algorithms are also getting introduced.

The aim of this study was to validate the accuracy and reproducibility of a model-based software for RT3DE and CMR against our golden standard threshold based CMR software which is more time consuming.

\section{Materials and methods}

A total of 24 healthy children (12 males) were prospectively enrolled into the study referred to our institute to collect volumetric CMR and realtime 3D echocardiographic (RT3DE) reference values. Each subject underwent both modalities to determine the enddiastolic volumes (EDV), endsystolic volumes (ESV), stroke volumes (SV) and ejection fractions (EF) of the LV. To avoid physiologic day-to-day variations in ventricular filling and to ensure identical conditions for both 
techniques CMR and RT3DE each subject was examined within 60 minutes in random order. The study was approved by the local Ethics Institutional Review Committee (local registration number 25/2005) and conforms to the principles of the Helsinki Declaration as well as German law. Informed, written consent was obtained from the children's parents or legally authorized representative.

Usefulness and applicability of the different evaluation tools in a routine clinical setting was tested in 11 patients with abnormally shaped left ventricles -2 pts with Tetralogy-of-Fallot, 4 pts with dilated cardiomyopathy, 1 pt with myocarditis, 1 Fontan pt and 3 pts with aortic stenosis and aortic insufficiency. Indication for CMR of patients were clinically arranged to estimate volume overload and the overall cardiac constitution prior secondary treatment.

\subsection{Realtime three-dimensional echocardiography}

For RT3DE a Vivid 7 ultrasound device equipped with a V3 matrix transducer (General Electric, Milwaukee, Wisc., USA) was used. Both, volunteers and patients, were examined in the left lateral decubitus position. The transducer with a footprint of $21 \times 26 \mathrm{~mm}$ was placed in the apical position with image axis aligned to the LV long axis. The acquisition of 4 subvolumes with a maximum FOV of $90^{\circ}$ was performed during a single end-expiratory breathhold to obtain one full volume data set with stable transducer position. Temporal resolution was 20-26 bps. To achieve best image quality for RT3DE the parameters of automatic tissue optimization and full speckle reduction were used in addition to the standard preset settings supplied by the manufacturer.

\subsection{Evaluation of RT3DE data}

RT3DE data sets were imported to the Research-Arena platform (TOMTEC, Version 2.0.0 Build 1.29, Unterschleissheim, Germany), subsequently quantification of volumes was performed using the model-based 4D LV-Analysis® tool (Version 2.5 Build 12), labelled as "SWT-echo", which is comparable to the aforementioned CMR evaluation software SWT-mri. After adjusting of the data set to obtain the largest long axis dimension in order to prevent foreshortening enddiastolic and endsystolic contours were tracked manually in 4-chamber, 2-chamber and apical long axis views (Figure 1). Trabeculations and papillary muscles were included in the LV-cavity. Functional LV parameters were calculated semi-automatically by the system as described in the instruction manual of the manufacturer. For ventricular border detection, a contour-finding activity of 30 intensity units was applied which had been proven as the most reliable value to achieve high accordance between CMR and RT3DE volumetric data ${ }^{[5]}$.
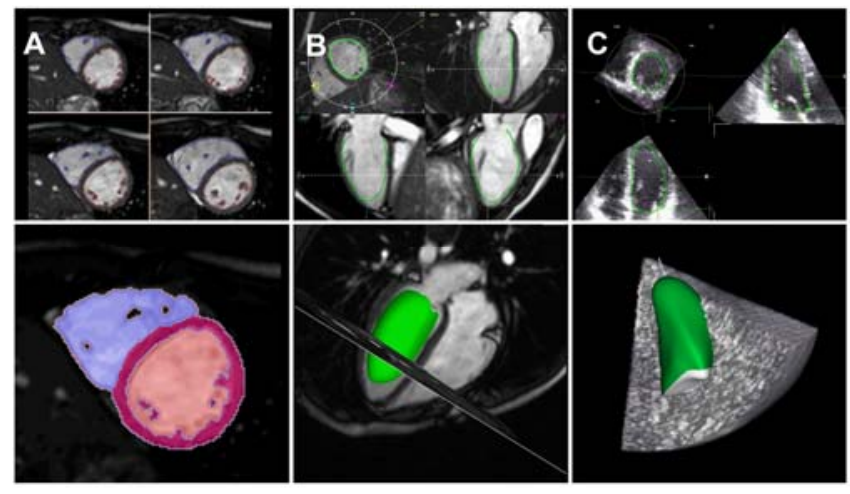

Figure 1. Representative data sets for volume quantification. (A) Non-model based SWP-mri. A subset of CMR enddiastolic short-axis slices depicting left (red) and right (blue) ventricular inner borders. Calculation of the ventricular volume by summation of the appropriate areas. (B) Model based 4D LV MR analysis (SWT-mri, TOMTEC). Manual tracking of LV borders in one two-, three- and four-chamber view (upper image), with successive fine-tuning in the short-axis stack to generate the moving 3D "Beutel". (C) Model-based 4D LV analysis tool for evaluation of RT3DE data sets (SWT-echo, TOMTEC). After adjusting of the data set to obtain the largest long axis dimension enddiastolic and endsystolic contours were tracked manually in four-, two-chamber and apical long axis views 


\subsection{Cardiovascular magnetic resonance imaging}

The children were placed supine onto the examination table. Vector electrocardiogram leads and an abdominal belt for detection of respiratory motion were attached. Volumetric CMR was performed on a 1.5T MR system (Philips Medical Systems, Best, The Netherlands, Intera, R12, maximum gradient performance $30 \mathrm{mT} / \mathrm{m}$, slew rate $150 \mathrm{~T} . \mathrm{m}-1 . \mathrm{s}-1$ ) with a 5 -element cardiac synergy surface coil for signal detection.

A short-axis multi-slice multi-phase ECG-triggered steady-state free precession gradient-echo (SSFP) sequence $\left(\mathrm{TR} / \mathrm{TE} / \mathrm{flip}=2.8 \mathrm{msecs} / 1.4 \mathrm{msecs} / 60^{\circ}\right)$ was applied to cover the entire cardiac chambers. Typically, 24 to 34 slices were obtained during 14 secs expiration breathhold maneuvers ( 2 slices per breathhold) with 20 heart phases per slice. Slice thickness was $6 \mathrm{~mm}$ with no slice gap, matrix size $160 \times 240$, field-of-view $370 \mathrm{~mm}$ resulting in an in-plane resolution of $2.3 \times 1.5 \mathrm{~mm}$ with the higher resolution in the phase encoding direction. A SENSE-reduction factor in phase-encoding direction of 2 was used.

An additional SSFP acquisition with a radial stack of 6 slices and an angulation of 30 degrees was introduced for volume quantification using the model-based 4D LV-Analysis MRC tool (TOMTEC, see below). All sequence parameters were kept identical except the higher field-of-view of $520 \mathrm{~mm}$.

\subsection{Evaluation of CMR data}

Quantification of the volumetric CMR data was performed offline

(1) on a workstation using the non-model and threshold-based "HDZ MR-Tools" software package (labeled as "SWP-mri"; HDZ, Bad Oeynhausen, Germany). A condensed description of the software package is found elsewhere ${ }^{[5]}$. To ensure compatibility to the model-based analysis packages, papillary muscles and trabecular structures were included exceptionally into the ventricular lumen by the software. No geometric model or assumption was needed for this evaluation tool. LV volumes were calculated by summation of the cavity areas multiplied with the slice thickness in all appropriate slices (Figure 1).

(2) On a personal computer using the Image Arena VA platform (TOMTEC, Unterschleissheim, Germany). LV volume quantification was performed with the model-based 4D LV-Analysis MRC tool (Version 1.0), labeled as "SWT-mri". After defining of the three obligatory LV long axes (2-, 3-, and 4-chamber views, radial acquisition stack), a short axis stack was loaded (see Figure 1). The enddiastolic and endsystolic contours of the endocardium were tracked manually in the three long axes views. Fine-tuning of the ventricular borders was performed in the short axis slices. Based on the LV model and the contour tracking a four-dimensional "Beutel" is calculated including trabeculations and papillary muscles. EDV, ESV and EF were calculated semi-automatically by the system as described in the instruction manual of the manufacturer.

\subsection{I n vitro validation}

Because true ventricular chamber dimensions are incapable of proof in vivo nine asymmetric agar agar phantoms ( $2 \%$ by weight) filled with known amounts of tap water (inner lumen: $20-350 \mathrm{ml}$ ) were prepared (Figure 2). All RT3DE and CMR measurements were performed three times within 3 hours of time to assess repeatability. The averaged values were regarded as reference values. To ensure comparability with the in vivo studies CMR was performed using identical imaging parameter settings of the applied SSFP pulse sequence as described above with a simulated heart rate of 80 beats-per-minute.

\subsection{Statistical analysis}

RT3DE and CMR data, respectively, were quantified each by a single expert. Descriptive statistics was obtained from all relevant continuous variables. Correlation analysis was done by linear regression analysis. Normal distribution was tested 
using the Kolmogorov-Smirnoff test. Analysis of Bland-Altman was performed to quantify the agreement between the different evaluation algorithms, to compare CMR vs. RT3DE volumetry and to assess intraobserver variability. Significance of the observed differences between the functional ventricular parameters EDV, ESV, SV and EF was tested with a two-sided paired Student's t-test. A P-value of $\leq 0.05$ was considered as statistically significant.

Figure 2. Asymmetric gel phantom. Steady-state free precision gradientecho CMR image. Representative asymmetric agar agar phantom containing fine structures for in vitro validation of non-model and modelbased evaluation software. Measured inner volume was $150 \mathrm{ml}$

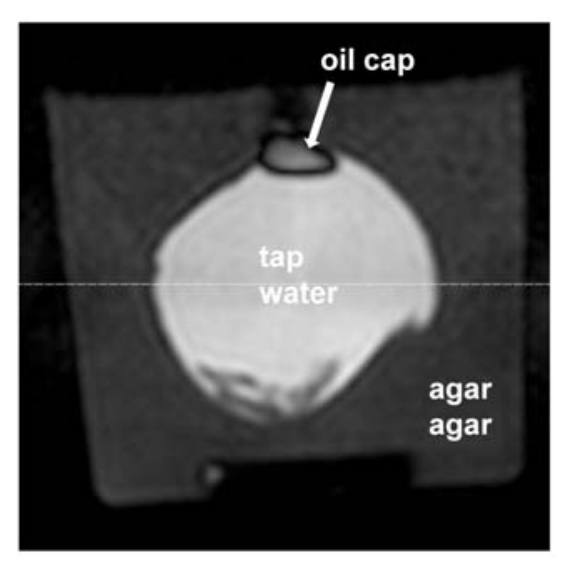

\section{Results}

\subsection{Phantom study}

The in vitro study with asymmetric agar agar phantoms revealed excellent agreement between reference data and volumetric data received by the non-model-based software package SWP-mri (Bland-Altman statistics: mean $\pm \mathrm{SD}=0.7 \pm$ $1.9 \%$, limits-of-agreement (LOA): $-3.2 \%$ to $4.5 \%$ ) with a maximal deviation from the "true" value of $4.6 \%$ (see Table 1 and Figure 3). Evaluation of the RT3DE data using the model-based SWT-echo resulted in a somewhat higher discrepancy from the references of below $8 \%$. Bland-Altman statistics revealed a comparable agreement but with a slightly increased scatter (-0.6 $\pm 4.0 \%$, LOA: $-8.7 \%$ to $7.5 \%)$. Reference data were underestimated up to $18 \%$ applying the model-based SWT-mri (-13.1 $\pm 2.9 \%$, LOA: $-18.9 \%$ to $-7.3 \%)$.

Table 1. In vitro calibration measurements

\begin{tabular}{|c|c|c|c|c|c|c|c|}
\hline & \multirow{2}{*}{$\begin{array}{l}\text { Graded cylinder } \\
\text { Volume [ml] }\end{array}$} & \multicolumn{2}{|l|}{ CMR -SW ${ }_{\text {P-mri }}$} & \multicolumn{2}{|l|}{ CMR -SW $_{\text {T-mri }}$} & \multicolumn{2}{|c|}{ RT3DE -SW T-echo } \\
\hline & & Volume $^{*}$ [ml] & $\Delta[\%]$ & Volume $^{*}[\mathrm{ml}]$ & $\Delta[\%]$ & Volume $^{*}$ [ml] & $\Delta[\%]$ \\
\hline 1 & 20.0 & $19.9 \pm 0.2$ & -0.5 & $17.7 \pm 0.3$ & -11.3 & $20.3 \pm 1.2$ & 1.3 \\
\hline 2 & 35.0 & $36.6 \pm 0.7$ & 4.6 & $31.9 \pm 0.4$ & -8.8 & $32.4 \pm 1.2$ & -7.5 \\
\hline 3 & 54.0 & $53.6 \pm 0.5$ & -0.7 & $46.7 \pm 0.6$ & -13.6 & $53.2 \pm 2.1$ & -1.5 \\
\hline 4 & 69.0 & $69.7 \pm 0.5$ & 1.0 & $60.7 \pm 0.2$ & -12.0 & $71.2 \pm 2.6$ & 3.1 \\
\hline 5 & 85.0 & $87.1 \pm 1.1$ & 2.5 & $76.8 \pm 0.5$ & -9.6 & $87.9 \pm 5.4$ & 3.5 \\
\hline 6 & 108.0 & $109.4 \pm 0.6$ & 1.3 & $93.7 \pm 2.9$ & -13.2 & $105.9 \pm 9.8$ & -1.9 \\
\hline 7 & 150.0 & $149.3 \pm 1.0$ & -0.5 & $132.4 \pm 2.0$ & -11.7 & $151.8 \pm 6.0$ & 1.2 \\
\hline 8 & 237.0 & $232.7 \pm 2.8$ & -1.8 & $195.4 \pm 2.5$ & -17.6 & $244.0 \pm 19.4$ & 2.9 \\
\hline \multirow[t]{2}{*}{9} & 315.0 & $315.7 \pm 1.5$ & 0.2 & $276.3 \pm 7.8$ & -12.3 & $297.0 \pm 2.1$ & -5.7 \\
\hline & mean $\pm \mathrm{SD}$ & & $0.7 \pm 1.9$ & & $-12.2 \pm 2.5$ & & $-0.5 \pm 4.0$ \\
\hline
\end{tabular}

Note. Validation of asymmetric agar agar phantom volumes. CMR data were evaluated using the non-model based SW (SWP-mri) and the model-based 4D LV-analysis MRCtool (SWT-mri), respectively. RT3DE volumes were determined by the model-based 4D LV-Analysis $®$ tool (SWT-echo). $\Delta=$ difference to graded cylinder volumes serving as reference. * mean values \pm standard deviation of three measurements demonstrating observer variability 
Figure 3. Phantom study

Bland-Altman plots (left) and linear regression plots (right) depicting conformity of measured volumes with volumetric values obtained with the different evaluation software packages. Upper row: non modelbased SWP-mri vs. graded cylinder; middle row: model-based SWT-mri vs. graded cylinder; lower row: model-based SWT -echo vs. graded cylinder
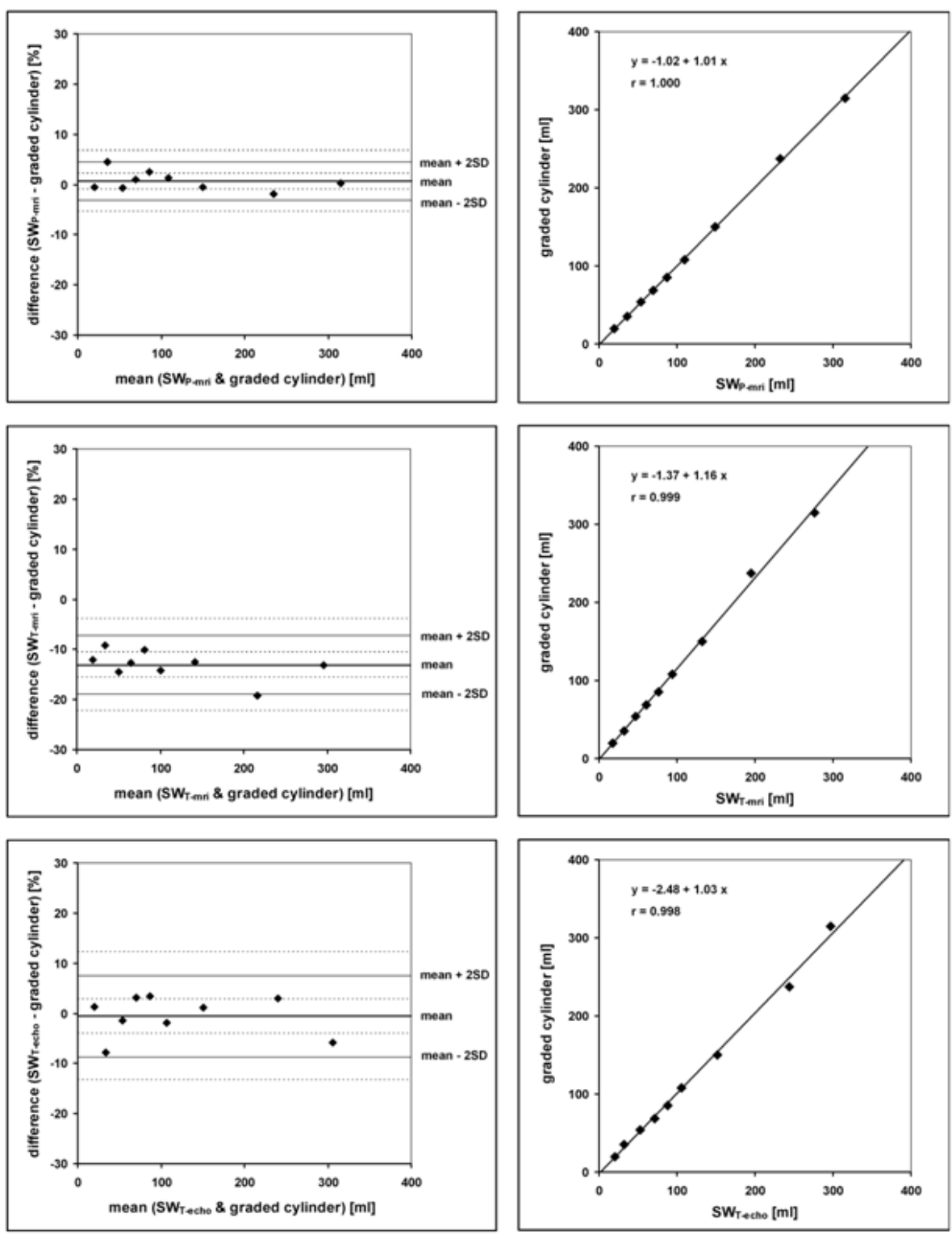

\subsection{Volunteers}

Mean age of the study population was $11.4 \pm 3.3$ years (range 5.3 to 18.8 years, median $=11.8$ years). Sinus rhythm was present in all participants. Mean heart rate was $88 \pm 14 \mathrm{bpm}$ (range 61 to $117 \mathrm{bpm}$, median $=86 \mathrm{bpm}$ ). All scans were completed successfully. RT3DE was completed within 10 minutes, volumetric CMR study was finished within 20 minutes of scan time.

Comparing RT3DE and CMR data only a marginal underestimation in EDV was observed but with slightly higher scatter as indicated by the LOA if the model-based software SWT-echo was related to the non-model based SWP-MRI (5.3 \pm 12.7 $\%, p=0.015$, Table 2$)$. A weak but statistically not relevant overestimation was detected for ESV $(-1.3 \pm 20.1 \%, p=$ 0.916). Consequently, mean ejection fraction was pronounced using CMR data $(4.0 \pm 8.0 \%, p=0.024)$. 
Table 2. Volunteers

\begin{tabular}{|c|c|c|c|c|c|c|c|c|c|}
\hline \multirow{2}{*}{ LOA } & \multicolumn{3}{|c|}{ SW $_{\text {P-mri }}$ Vs. SW T-mri } & \multicolumn{3}{|c|}{ SW $_{\text {P-mri }}$ VS. $S W_{\text {T-echo }}$} & \multicolumn{3}{|c|}{ SW $_{\text {T-echo }}$ VS. SW $\mathbf{S W}_{\text {T-mri }}$} \\
\hline & EDV & ESV & EF & EDV & ESV & EF & EDV & ESV & EF \\
\hline $\mathrm{N}$ & 24 & 24 & 24 & 24 & 24 & 24 & 24 & 24 & 24 \\
\hline mean & 15.1 & -11.0 & 16.6 & 5.3 & -1.3 & 4.0 & 9.8 & -9.6 & 12.6 \\
\hline mean $+2 \mathrm{SD}$ & 27.9 & 24.1 & 35.4 & 30.8 & 38.9 & 19.9 & 40.0 & 41.0 & 34.9 \\
\hline mean - 2SD & 2.4 & -46.1 & -2.2 & -20.1 & -41.6 & -12.0 & -20.5 & -60.1 & -9.6 \\
\hline $\mathrm{r}$ & 0.990 & 0.901 & & 0.939 & 0.862 & & 0.936 & 0.801 & \\
\hline p-value & $<0.001$ & $<0.003$ & $<0.001$ & 0.015 & 0.916 & 0.024 & 0.021 & 0.020 & $<0.001$ \\
\hline
\end{tabular}

Note. Bland-Altman statistics of volumetric CMR data using the non-model based SW (SWP-mri) and the model-based 4D LV-analysis MRCtool (SWT-mri). For comparison RT3DE data were determined by the model-based 4D LV-Analysis $®$ tool (SWT-echo). Except the correlation coefficient and P-values all values are given in percent. Mean corresponds to mean difference. EDV = end diastolic volume; $\mathrm{ESV}=$ end systolic volume; $\mathrm{EF}=$ ejection fraction; $\mathrm{LOA}=$ limits-of-agreement; $\mathrm{r}=$ correlation coefficient.

Assessment of LV parameters using the same CMR data set but applying different evaluation strategies (non-model based SWP-MRI vs. model-based quantification software SWT-MRI), a moderate mean underestimation was observed for the model-based SWT-MRI for EDV (mean \pm SD: $15.1 \pm 6.4 \%$ ) and a mean overestimation for ESV $(-11.0 \pm 17.5 \%)$ as indicated by Bland-Altman analysis (see Table 2, Figure 4) leading to an overestimation of EF (16.6 $\pm 18.8 \%)$. All differences were statistically significant. Strong correlation was found for both EDV and ESV $(r>0.900)$, respectively.

Figure 4. Healthy subjects

Comparison of CMR enddiastolic volumes assessed by non-model based contour finding algorithm (SWP-MRI) vs. model based 4D LV analysis MR algorithm (SWT-MRI). Volumetric indices were compared with realtime $3 \mathrm{D}$ echocardiography applying a similar "Beutel" algorithm (SWTecho). Bland-Altman (left column) and linear correlation plots (right column)
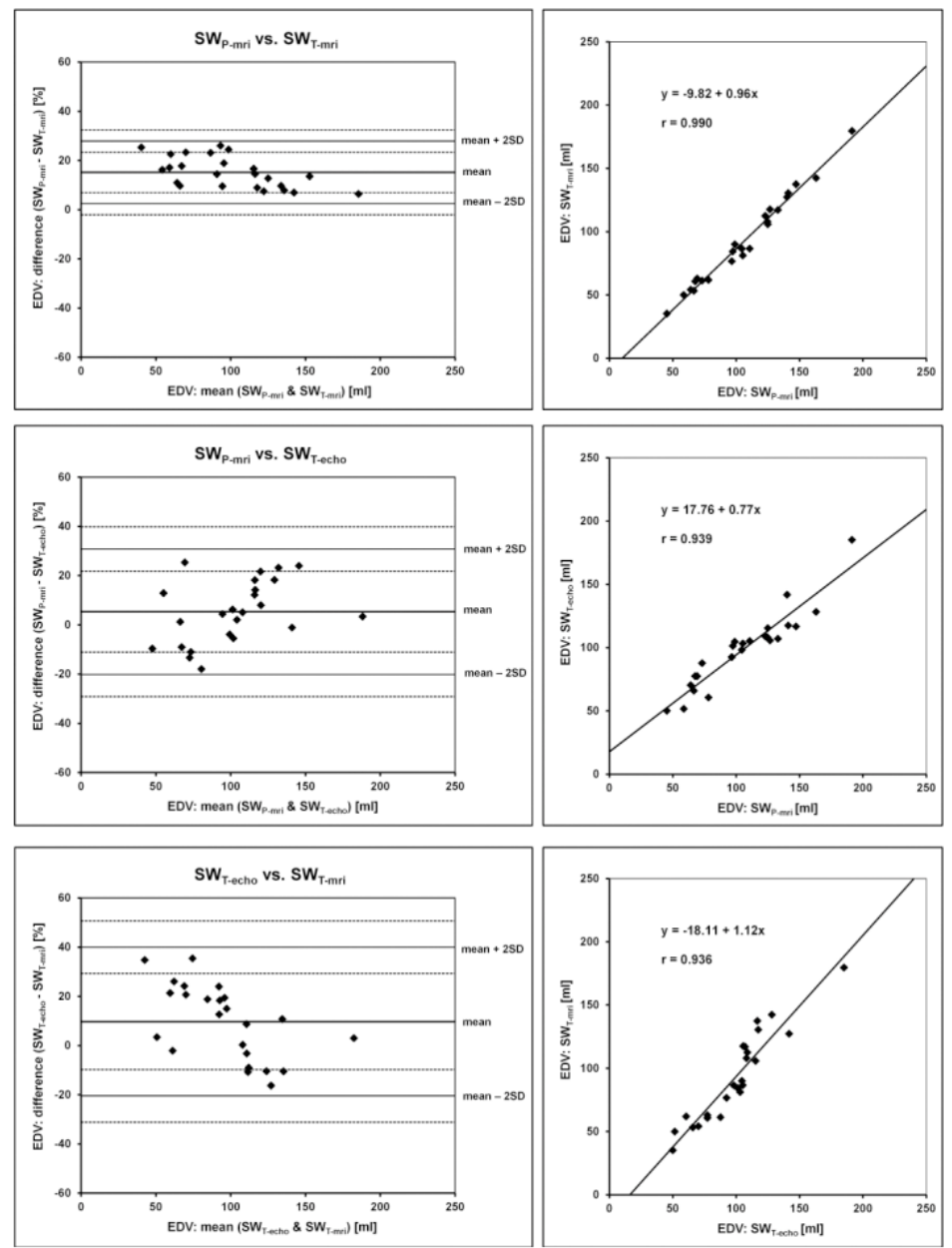
If the model-based MR software SWT-mri was compared with the model-based echocardiographic software SWT-echo a moderate underestimation for EDV $(9.8 \pm 15.1 \%)$ and EF $(12.6 \pm 11.1 \%)$ and a moderate overestimation for ESV $(-9.6 \pm$ $25.3 \%)$ with a higher scatter was detected with the former quantification software. All differences were statistically significant.

\subsection{I ntraobserver agreement}

Intraobserver agreement was high with a low scatter using the non-model based SWP-mri (Bland-Altman: 1\%, SD: $\sim 5 \%$ ). A marginal weaker agreement was found for the two model-based quantification software packages SWT-mri and SWT-echo, respectively, including a slightly increased scatter ( 4\%, SD between $6-15 \%$, Table 3).

Table 3. Intraobserver variability

\begin{tabular}{|c|c|c|c|c|c|c|c|c|c|}
\hline \multirow{2}{*}{ LOA } & \multicolumn{3}{|c|}{ SW $_{\text {P-mri }}$} & \multicolumn{3}{|c|}{$\mathrm{SW}_{\mathrm{T}-\mathrm{mri}}$} & \multicolumn{3}{|c|}{ SW $_{\text {T-echo }}$} \\
\hline & EDV & ESV & EF & EDV & ESV & EF & EDV & ESV & EF \\
\hline $\mathrm{N}$ & 13 & 13 & 13 & 12 & 12 & 12 & 24 & 24 & 24 \\
\hline mean & -0.7 & 1.0 & -1.1 & 4.3 & 0.6 & 2.3 & 3.8 & 0.0 & 2.0 \\
\hline mean $+2 \mathrm{SD}$ & 7.9 & 11.8 & 4.0 & 20.1 & 26.8 & 14.2 & 20.8 & 30.4 & 20.5 \\
\hline mean - 2SD & -9.4 & -9.9 & -6.2 & -11.4 & -25.6 & -9.7 & -13.2 & -30.3 & -16.5 \\
\hline$r$ & 0.996 & 0.995 & & 0.990 & 0.979 & & 0.958 & 0.931 & \\
\hline$p$-value & 0.861 & 0.473 & 0.165 & 0.118 & 0.556 & 0.218 & 0.051 & 0.775 & 0.312 \\
\hline
\end{tabular}

Note. Bland-Altman statistics of volumetric CMR data using the non-model based SW (SWP-mri) and the model-based 4D LV-analysis MROtool (SWT-mri). For comparison intraobserver variability of RT3DE data was determined by the model-based 4D LV-Analysis $®$ tool (SWT-echo). Except the correlation coefficient and P-values all values are given in percent. Mean corresponds to mean difference. $\mathrm{EDV}=$ end diastolic volume; $\mathrm{ESV}=$ end systolic volume; $\mathrm{EF}=$ ejection fraction; $\mathrm{LOA}=$ limits-of-agreement; $\mathrm{r}=$ correlation coefficient.

\subsection{Patients with abnormally shaped left ventricles}

Mean age of the patient group was $22.0 \pm 17.0$ years (range 8.2 to 65.2 years, median $=15.7$ years). Sinus rhythm was present in all subjects during the examination. Mean heart rate was $73 \pm 16 \mathrm{bpm}$ (range 51 to $99 \mathrm{bpm}$, median $=75 \mathrm{bpm}$ ). Volumetric RT3DE and CMR data were gathered during clinical routine examinations with comparable expenditure of time as was needed for volunteers.

Using SWP-mri enddiastolic left ventricular volumes in patients with abnormally shaped cardiac chambers ranged from $57 \mathrm{ml}$ to $404 \mathrm{ml}$ and endsystolic volumes from $29 \mathrm{ml}$ to $293 \mathrm{ml}$. Quantification with RT3DE revealed an underestimation of $\operatorname{EDV}(11.5 \pm 15.0 \%, p<0.05)$ and $\operatorname{ESV}(13.0 \pm 9.2 \%, p<0.05)$. In analogy $\operatorname{EDV}(12.1 \pm 7.1 \%, p<0.05)$ and $\operatorname{EF}(2.4$ $\pm 13.1 \%, p=0.686$ ) were underestimated by using the model-based SWT-mri (reference $=$ SWP-mri), whereas - in contrary to the volunteer study - ESV $(10.6 \pm 15.9 \%, p<0.05)$ was underestimated as well (see Table 4, Figure 5).

Table 4. Patients with abnormally shaped ventricles

\begin{tabular}{|c|c|c|c|c|c|c|c|c|c|}
\hline \multirow{2}{*}{ LOA } & \multicolumn{3}{|c|}{ SW $_{\text {P-mri }}$ VS. $S_{\text {T-mri }}$} & \multicolumn{3}{|c|}{$S_{\text {P-mri }}$ VS. SW $W_{\text {T-echo }}$} & \multicolumn{3}{|c|}{ SW $_{\text {T-echo }}$ VS. SW $\mathbf{S W}_{\text {T-mri }}$} \\
\hline & EDV & ESV & $\mathbf{E F}$ & EDV & ESV & $\mathbf{E F}$ & EDV & ESV & $\mathbf{E F}$ \\
\hline $\mathrm{N}$ & 11 & 11 & 11 & 11 & 11 & 11 & 11 & 11 & 11 \\
\hline mean & 12.1 & 10.6 & 2.4 & 11.5 & 13.0 & -6.9 & 0.6 & -2.4 & 9.3 \\
\hline mean $+2 \mathrm{SD}$ & 26.4 & 42.4 & 28.5 & 41.4 & 31.5 & 36.1 & 29.4 & 33.4 & 54.3 \\
\hline mean - 2SD & -2.1 & -21.2 & -23.7 & -18.5 & -5.4 & -49.9 & -28.2 & -38.2 & -35.7 \\
\hline $\mathrm{r}$ & 0.992 & 0.994 & & 0.964 & 0.997 & & 0.969 & 0.994 & \\
\hline p-value & $<0.001$ & 0.026 & 0.686 & 0.026 & 0.018 & 0.441 & 0.561 & 0.155 & 0.297 \\
\hline
\end{tabular}

Note. Bland-Altman statistics of volumetric CMR data using the non-model based SW (SWP-mri) and the model-based 4D LV-analysis MROtool (SWT-mri). For comparison RT3DE data were determined by the model-based 4D LV-Analysis $®$ tool (SWT-echo). Except the correlation coefficient and P-values all values are given in percent. Mean corresponds to mean difference. EDV = end diastolic volume; $\mathrm{ESV}=$ end systolic volume; $\mathrm{EF}=$ ejection fraction; $\mathrm{LOA}=$ limits-of-agreement; $r$ = correlation coefficient. 
Figure 5. Patients with asymmetrical shaped left ventricles. Comparison of CMR enddiastolic volumes assessed by non-model based contour finding algorithm (SWP-mri) vs. model based 4D LV analysis MR algorithm (SWT-mri). Volumetric indices were compared with realtime 3D echocardiography applying a similar "Beutel" algorithm (SWT-echo). BlandAltman (left column) and linear correlation plots (right column)
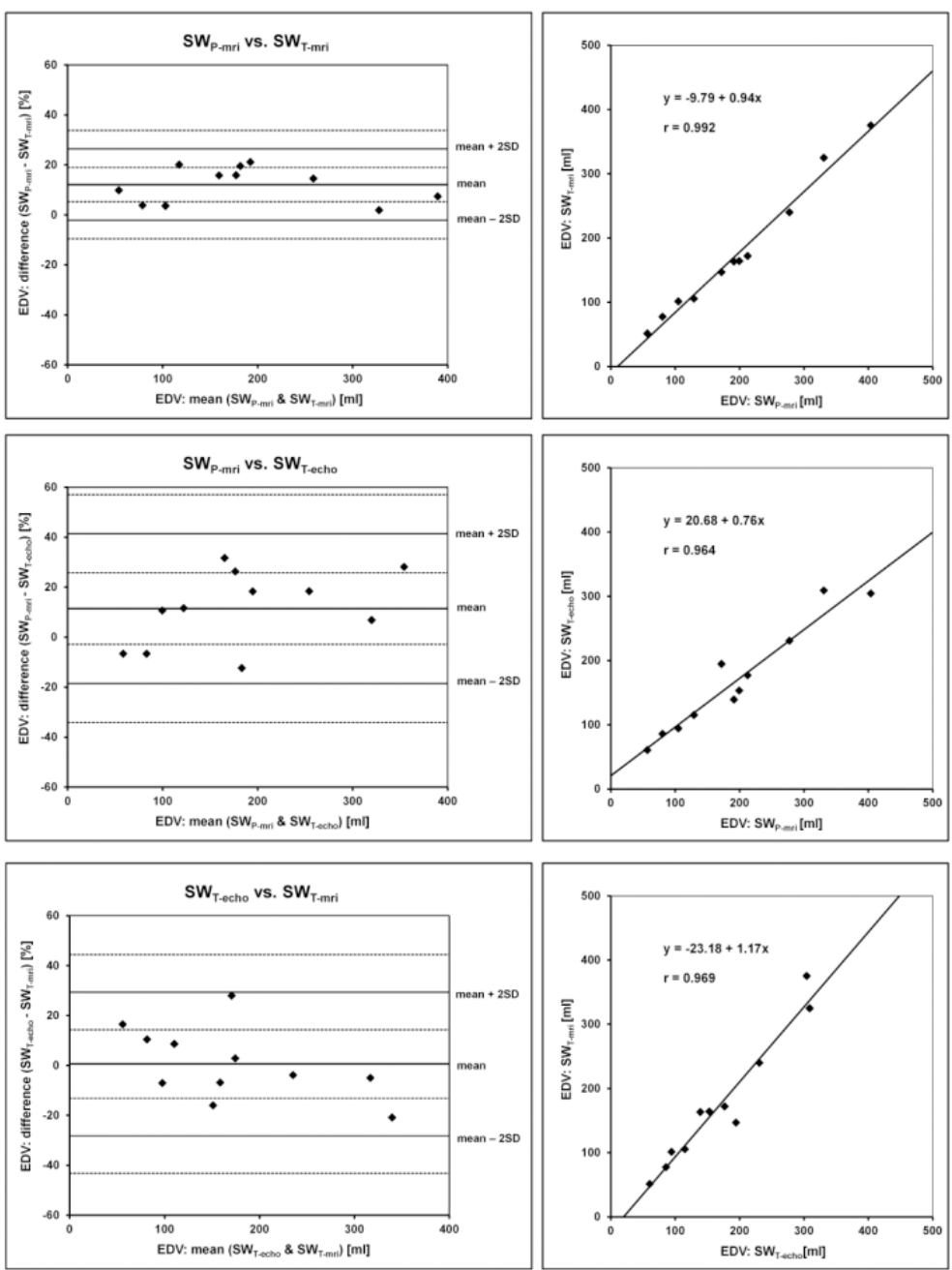

\section{Discussion}

Global and regional changes in cardiac function caused by congenital or acquired heart disease ${ }^{[21]}$ such as coarctation, aortic stenosis or cardiomyopathy as well as remodeling processes after surgical or interventional therapy are the diagnostic domain of non-invasive tools such as echocardiography and CMR. They can be assessed by tissue deformation imaging using speckle tracking, CMR tagging ${ }^{[22]}$ as well as by three dimensional techniques to measure changes in blood pool volumes throughout the cardiac cycle. With the introduction of model-based analysis algorithms motional details such as parametric maps for dynamic visualization of contraction time or assessment of mechanical dyssynchrony are now accessible ${ }^{[7,16,23,24]}$ which are easy and quick to apply but accuracy and reliability of functional LV parameters still have to be investigated further. It was the aim of this study to consolidate the promising volumetric results in healthy pediatric individuals assessed by RT3DE in a former study ${ }^{[5]}$ by examining asymmetrically shaped gel phantoms and a patient group with predominantly dilated left ventricles. Furthermore we wanted to test if the same methodological approach of model-based volumetry can be used for CMR volumetry in order to save time for postprocessing and take advantage of the accessory possibilities resulting in quantification of regional function and dyssynchrony later on.

To cover as far as possible all ventricular sizes typically found in small children up to patients with dilated LV chambers gel phantom volumes were designed ranging between 20 to $315 \mathrm{ml}$ with asymmetrical shapes to mimic excessive pressure or volume overload in congenital or acquired heart diseases ${ }^{[25]}$. The results of our gel phantom experiments revealed that volumes calculated with the non model-based SWP-mri agreed best with the graded cylinder volumes (see Table 1). 
RT3DE showed negligibly less agreement because of slightly higher scatters whereas we saw some greater volume underestimation of 10-15\% for SWT-mri. We hypothesize that the use of fixed contour finding border detection in the model-based SWT-mri tool was responsible for that because SWT-echo was quite precise using low contour finding activity values CFA (see Table 2). As pointed out in a meta-analysis by Shimada et al. ${ }^{[26]}$ a multiplicity of previously published work revealed underestimation of LV volumes by RT3DE compared to CMR with significant heterogeneity among different studies. Furthermore, the existence of cardiac disease was associated with increased underestimation. A similar behaviour was observed in the present study where underestimation of LV volumes in patients with abnormally shaped and predominantly dilated LV's was present as well. In volunteers only enddiastolic volumes were slightly underestimated by RT3DE whereas it was not obvious in endsystolic volumes and in the phantom study. Adjustments of the contour finding activity value which had been proven to be more precise in the definition of endocardial ventricular borders ${ }^{[5]}$ in combination with the underlying 4D LV model might be responsible for improved accordance in these cases. Possibly the underlying model fits better with normal LV shape independently from contour finding activity. If the new speckle tracking based RT3DE tools are more precise in the assessment of these dilated chambers remains to be investigated further. It has to be emphasized that the amount of volume underestimation as well as the scatters are excellent for RT3DE volumetry applying a matrix probe constructed for the use in adults to children.

Intraobserver variability for LV functional parameters was low for the non model-based SWP-mri and was comparable with the literature ${ }^{[5,17]}$. Whereas Bland-Altman statistics revealed high agreement for the model-based evaluation routines both for CMR as well as for echocardiographical data a significantly elevated scatter expressed by the LOA was obvious. This could be explained by the increased observer dependence and beat to beat variations of volumes for echocardiographical data ${ }^{[4,27]}$ but not sufficiently for the present CMR data sets which were identical for both CMR evaluation tools, SWP-mri and SWT-mri, respectively. As shown in Figure 1 the semi-automatic algorithm for generation of the moving four-dimensional "Beutel" model using SWT-mri which is based on a predefined LV model in conjunction with manual defined contour tracking points as start condition occasionally failed to precisely match the endomyocardial contours in the apical ventricular area and in non-uniform cardiac sections (which occur frequently in CHD where cardiac deformations are common). This is possibly induced by extensive smoothing during generation of the LV model which prevents a more dominant use of the manual contour tracking spline points. Therefore, underestimation of enddiastolic volumes in volunteers and patients, respectively, compared to the non model-based version have to be expected.

Surprisingly, larger endsystolic volumes were found with SWT-mri in healthy subjects but not in our patients with enlarged ventricular chambers. This could be explained by the fact that endocardial borders in systolic heart phases in our volunteers were occasionally overestimated (see Figure 6) when contour revision of the four-dimensional "Beutel" was performed with the tracking optimization routine proposed by the manufacturer to keep workflow easy and time efficient and acceptable for clinical routine. (The tracking optimization allows the user to modify the "Beutel" only at the enddiastole whereas the recalculation of the 4D model in all phases and slices is done by the system.). In our patients with an excessive pressure or volume overload on the LV this overestimation was not obvious because of the extended endsystolic volumes which were initiated by the patient's disease. As shown in Tables 5a and b mean left ventricular ESV in patients were comparable to mean EDV in volunteers leading to an improved detection of the ventricular shape during recalculation by the system. Consequently, the calculated EF did not differ considerably between both techniques in the patient group but was significantly lower $(\sim 15 \%)$ in healthy volunteers applying SWT-mri.

Table 5a. Healthy subjects: Mean left ventricular volumes and EF

\begin{tabular}{llll}
\hline & Healthy subjects & EF [\%] \\
\cline { 2 - 4 } & EDV [ml] & ESV [ml] & $65.8 \pm 4.9$ \\
\hline $\mathrm{SW}_{\text {P-mri }}$ & $106.4 \pm 36.7$ & $36.3 \pm 13.6$ & $55.7 \pm 4.6$ \\
$\mathrm{SW}_{\text {T-mri }}$ & $92.6 \pm 35.7$ & $41.0 \pm 15.9$ & $63.3 \pm 5.8$ \\
$\mathrm{SW}_{\text {T-echo }}$ & $99.2 \pm 29.9$ & $36.1 \pm 11.6$ & \\
\hline
\end{tabular}

Note. Functional LV parameters according to the applied evaluation software for CMR and RT3DE data.

Published by Sciedu Press 
Table 5b. Patients with abnormally shaped LV: Mean left ventricular volumes and EF

\begin{tabular}{llcr}
\hline & Patients & & \\
\cline { 2 - 4 } & EDV [ml] & ESV [ml] & EF [\%] \\
\hline SW $_{\text {P-mri }}$ & $196.3 \pm 107.0$ & $115.7 \pm 97.2$ & $46.3 \pm 17.9$ \\
SW $_{\text {T-mri }}$ & $174.7 \pm 101.4$ & $107.5 \pm 97.1$ & $45.6 \pm 18.1$ \\
SW $_{\text {T-echo }}$ & $169.4 \pm 84.1$ & $97.0 \pm 76.3$ & $47.8 \pm 15.4$ \\
\hline
\end{tabular}

Note. Functional LV parameters according to the applied evaluation software for CMR and RT3DE data.

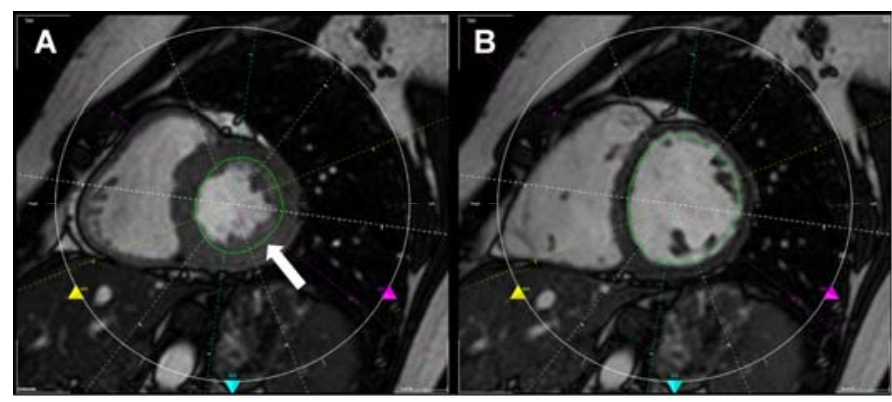

Figure 6. Border detection impreciseness. (A) Illustration of endsystolic ventricular lumen overestimation with SWT-mri after revision of the four-dimensional "Beutel" which was performed with the manufacturer's tracking optimization routine. (B) Except for the apical slices enddiastolic endomyocardial borders were detected successfully

Left ventricular muscle mass was accessible for CMR data with SWP-mri and SWT-mri, respectively, whereas determination was not possible for RT3DE data. Furthermore, the total ventricular size was calculated. Complementary to the enddiastolic volumes muscle mass was overestimated by the model-based version (see Table 6) with high statistical significance $(p=0.006)$ in both study groups. Otherwise, because total left ventricular size represents the sum of enddiastolic blood volume and muscle mass agreement of both evaluation tools was high for this parameter and differences were not statistically significant any more. This further underscores that the restrictive guidelines for calculation of the 4D "Beutel" might be responsible for underestimation of enddiastolic lumen borders.

Table 6. left ventricular muscle mass and total ventricular size

\begin{tabular}{|c|c|c|c|c|c|}
\hline & \multirow{2}{*}{ 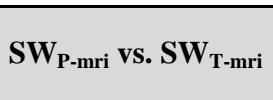 } & \multicolumn{2}{|c|}{ volunteers } & \multicolumn{2}{|c|}{ patients } \\
\hline & & $\mathbf{M M}$ & $\mathbf{V}_{\text {total }}$ & $\mathbf{M M}$ & $V_{\text {total }}$ \\
\hline \multirow{6}{*}{ LOA } & $\mathrm{N}$ & 24 & 24 & 11 & 11 \\
\hline & mean & -14.9 & 1.4 & -15.0 & 0.5 \\
\hline & mean $+2 \mathrm{SD}$ & 12.6 & 11.0 & -1.0 & 9.8 \\
\hline & mean - 2SD & -42.4 & -8.1 & -29.0 & -8.7 \\
\hline & $\mathrm{r}$ & 0.947 & 0.991 & 0.991 & 0.998 \\
\hline & p-value & 0.006 & 0.202 & 0.006 & 0.327 \\
\hline
\end{tabular}

Note. Bland-Altman statistics of left ventricular muscle mass and total ventricular size accessible by CMR data using the non-model based SW (SWP-mri) and the model-based 4D LV-analysis MRCtool (SWT-mri). No corresponding data were available by RT3DE. Except the correlation coefficient and P-values all values are given in percent. Mean corresponds to mean difference. MM = left ventricular muscle mass; Vtotal $=$ total ventricular size $(\mathrm{EDV}+\mathrm{MM}) ; \mathrm{LOA}=$ limits-of-agreement; $r=$ correlation coefficient

\section{Limitations}

In this study only intraobserver variability was determined because in preceding work intraobserver variability, interobserver variability and interexamination variability was already published for SWP-mri and SWT-echo ${ }^{[5,17]}$.

Although graded cylinder volumes were considered as the best approximation to the "true" volumes some inaccuracies may occur due to measuring tolerance of the applied syringes (systematic errors) and reading errors by the observer 
(statistical errors). Furthermore investigation of moving phantoms by RT3DE and CMR would have been of accessory value.

No conclusions were rendered in the presented study addressing disorders in the cardiac motion. It was the scope of this study to estimate in a first step the accuracy of different evaluation strategies to provide a basis for a better interpretation of motional parameters in future work.

\section{Conclusion}

Regarding accuracy and reliability RT3DE volumetry using the model-based approach and low CFA showed excellent results compared to the gold standard CMR using a semiautomatic non-model based technique in gel phantoms and healthy volunteers. Slight volume underestimation has to be accepted in dilated abnormally shaped ventricles. These results cannot be assigned similarly to the equally designed model based CMR evaluation software because of a less control on contour finding and especially endsystolic volumes by the operator. Critical validation of new tools is mandatory before implementation into the clinical workflow especially in the field of pediatric LV volumetry.

\section{Conflict of interest}

The authors declare that they have no conflict of interest.

\section{Acknowledgements}

We express our thanks to Gabriele Uges and Jessica Garen for organization and medical attendance of the patients.

\section{References}

[1] White HD, Norris RM, Brown MA, Brandt PW, Whitlock RM, Wild CJ. Left ventricular end-systolic volume as the major determinant of survival after recovery from myocardial infarction. Circulation 1987; 76:44-51. PMid:3594774 http://dx.doi.org/10.1161/01.CIR.76.1.44

[2] Chukwu EO, Barasch E, Mihalatos DG, Katz A, Lachmann J, Han J, et al. Relative importance of errors in left ventricular quantitation by two-dimensional echocardiography: Insights from three-dimensional echocardiography and cardiac magnetic resonance imaging. J Am Soc Echocardiogr 2008; 21:990-997. PMid:18765174 http://dx.doi.org/10.1016/j.echo.2008.07.009

[3] Pennell D, Sechtem U, Higgins C, Manning W, Pohost G, Rademakers F, et al. Clinical indications for cardiovascular magnetic resonance (cmr): Consensus panel report. Eur Heart J 2004; 25:1940-1965. PMid:15522474 http://dx.doi.org/10.1016/j.ehj.2004.06.040

[4] Grothues F, Smith GC, Moon JC, Bellenger NG, Collins P, Klein HU, et al. Comparison of interstudy reproducibility of cardiovascular magnetic resonance with two-dimensional echocardiography in normal subjects and in patients with heart failure or left ventricular hypertrophy. Am J Cardiol 2002; 90:29-34. http://dx.doi.org/10.1016/S0002-9149(02)02381-0

[5] Laser K, Bunge M, Hauffe P, Argueta J, Kelter-Klopping A, Barth P, et al. Left ventricular volumetry in healthy children and adolescents: Comparison of two different real-time three-dimensional matrix transducers with cardiovascular magnetic resonance [in process citation]. Eur J Echocardiogr 2010; 11:138-148. PMid:20019027 http://dx.doi.org/10.1093/ejechocard/jep185

[6] Jenkins C, Bricknell K, Hanekom L, Marwick T. Reproducibility and accuracy of echocardiographic measurements of left ventricular parameters using real-time three-dimensional echocardiography. J Am Coll Cardiol 2004; 44:878-886. PMid:15312875 http://dx.doi.org/10.1016/j.jacc.2004.05.050

[7] Frangi AF, Niessen WJ, Viergever MA. Three-dimensional modeling for functional analysis of cardiac images: A review. IEEE Trans Med Imaging 2001; 20:2-25. PMid:11293688 http://dx.doi.org/10.1109/42.906421

[8] Nassenstein K, de Greiff A, Hunold P. Mr evaluation of left ventricular volumes and function: Threshold-based 3d segmentation versus short-axis planimetry. Invest Radiol 2009; 44:635-640. PMid:19724238 http://dx.doi.org/10.1097/RLI.0b013e3181a9aaaf

[9] Moroseos T, Mitsumori L, Kerwin WS, Sahn DJ, Helbing WA, Kilner PJ, et al. Comparison of simpson's method and three-dimensional reconstruction for measurement of right ventricular volume in patients with complete or corrected transposition of the great arteries. Am J Cardiol 2010; 105:1603-1609. PMid:20494670 http://dx.doi.org/10.1016/j.amjcard.2010.01.025 
[10] Li B, Liu Y, Occleshaw CJ, Cowan BR, Young AA. In-line automated tracking for ventricular function with magnetic resonance imaging. JACC Cardiovasc Imaging 2010; 3:860-866. PMid:20705268 http://dx.doi.org/10.1016/j.jcmg.2010.04.013

[11] Codella NC, Cham MD, Wong R, Chu C, Min JK, Prince MR, et al. Rapid and accurate left ventricular chamber quantification using a novel cmr segmentation algorithm: A clinical validation study. J Magn Reson Imaging 2010; 31:845-853. PMid:20373428 http://dx.doi.org/10.1002/jmri.22080

[12] Feng W, Nagaraj H, Gupta H, Lloyd SG, Aban I, Perry GJ, et al. A dual propagation contours technique for semi-automated assessment of systolic and diastolic cardiac function by cmr. J Cardiovasc Magn Reson 2009; 11:30. PMid:19674481 http://dx.doi.org/10.1186/1532-429X-11-30

[13] Mazonakis M, Grinias E, Pagonidis K, Tziritas G, Damilakis J. Development and evaluation of a semiautomatic segmentation method for the estimation of $1 \mathrm{v}$ parameters on cine mr images. Phys Med Biol 2010; 55:1127-1140. PMid:20107252 http://dx.doi.org/10.1088/0031-9155/55/4/015

[14] Kleijn SA, Aly MF, Terwee CB, van Rossum AC, Kamp O. Reliability of left ventricular volumes and function measurements using three-dimensional speckle tracking echocardiography. Eur Heart J Cardiovasc Imaging 2012; 13:159-168. PMid:21926118 http://dx.doi.org/10.1093/ejechocard/jer174

[15] Riehle TJ, Mahle WT, Parks WJ, Sallee D, 3rd, Fyfe DA. Real-time three-dimensional echocardiographic acquisition and quantification of left ventricular indices in children and young adults with congenital heart disease: Comparison with magnetic resonance imaging. J Am Soc Echocardiogr 2008; 21:78-83. PMid:17628400 http://dx.doi.org/10.1016/j.echo.2007.05.021

[16] Hascoet S, Brierre G, Caudron G, Cardin C, Bongard V, Acar P. Assessment of left ventricular volumes and function by real time three-dimensional echocardiography in a pediatric population: A tomtec versus qlab comparison. Echocardiography 2010; 27:1263-1273. PMid:20584067 http://dx.doi.org/10.1111/j.1540-8175.2010.01235.x

[17] Sarikouch S, Peters B, Gutberlet M, Leismann B, Kelter-Kloepping A, Koerperich H, et al. Sex-specific pediatric percentiles for ventricular size and mass as reference values for cardiac mri: Assessment by steady-state free-precession and phase-contrast mri flow. Circ Cardiovasc Imaging 2010; 3:65-76. PMid:19820203 http://dx.doi.org/10.1161/CIRCIMAGING.109.859074

[18] Papavassiliu T, Kuhl HP, Schroder M, Suselbeck T, Bondarenko O, Bohm CK, et al. Effect of endocardial trabeculae on left ventricular measurements and measurement reproducibility at cardiovascular $\mathrm{mr}$ imaging. Radiology 2005; 236:57-64. PMid:15955850 http://dx.doi.org/10.1148/radiol.2353040601

[19] Hubka M, Bolson EL, McDonald JA, Martin RW, Munt B, Sheehan FH. Three-dimensional echocardiographic measurement of left and right ventricular mass and volume: In vitro validation. Int J Cardiovasc Imaging 2002; 18:111-118. PMid:12108906 http://dx.doi.org/10.1023/A:1014616603301

[20] Corsi C, Veronesi F, Lamberti C, Bardo DM, Jamison EB, Lang RM, et al. Automated frame-by-frame endocardial border detection from cardiac magnetic resonance images for quantitative assessment of left ventricular function: Validation and clinical feasibility. J Magn Reson Imaging 2009; 29:560-568. PMid:19243037 http://dx.doi.org/10.1002/jmri.21681

[21] Heimdal A, Stoylen A, Torp H, Skjaerpe T. Real-time strain rate imaging of the left ventricle by ultrasound. J Am Soc Echocardiogr 1998; 11:1013-1019. http://dx.doi.org/10.1016/S0894-7317(98)70151-8

[22] Hamdan A, Thouet T, Kelle S, Wellnhofer E, Paetsch I, Gebker R, et al. Strain-encoded mri to evaluate normal left ventricular function and timing of contraction at 3.0 tesla. J Magn Reson Imaging 2009; 29:799-808. PMid:19306402 http://dx.doi.org/10.1002/jmri.21684

[23] Koos R, Neizel M, Schummers G, Krombach GA, Stanzel S, Gunther RW, et al. Feasibility and initial experience of assessment of mechanical dyssynchrony using cardiovascular magnetic resonance and semi-automatic border detection. J Cardiovasc Magn Reson 2008; 10:49. PMid:18983646 http://dx.doi.org/10.1186/1532-429X-10-49

[24] Kapetanakis S, Kearney MT, Siva A, Gall N, Cooklin M, Monaghan MJ. Real-time three-dimensional echocardiography. A novel technique to quantify global left ventricular mechanical dyssynchrony. Circulation 2005; 112:992-1000. PMid:16087800 http://dx.doi.org/10.1161/CIRCULATIONAHA.104.474445

[25] Szathmary V, Ruttkay-Nedecky I. Computer model study of electrocardiologic manifestations in asymmetric left ventricular hypertrophy. J Cardiovasc Electrophysiol 2006; 17:1020-1025. PMid:16948748 http://dx.doi.org/10.1111/j.1540-8167.2006.00578.x

[26] Shimada YJ, Shiota T. A meta-analysis and investigation for the source of bias of left ventricular volumes and function by three-dimensional echocardiography in comparison with magnetic resonance imaging. Am J Cardiol 2011; 107: 126-138. PMid:21146700 http://dx.doi.org/10.1016/j.amjcard.2010.08.058

[27] Bellenger NG, Grothues F, Smith GC, Pennell DJ. Quantification of right and left ventricular function by cardiovascular magnetic resonance. Herz 2000; 25:392-399. PMid:10948775 http://dx.doi.org/10.1007/s000590050031 